\title{
Prosodic changes in aphasic speech: Timing
}

Article in Clinical Linguistics \& Phonetics · February 2010

DOI: $10.3109 / 02699200903464439 \cdot$ Source: PubMed

CITATIONS

4

4 authors, including:

Diana Van Lancker Sidtis

New York University

159 PUBLICATIONS $\quad$ 4,274 CITATIONS

SEE PROFILE

Catherine Jackson

California State University, Northridge

20 PUBLICATIONS 561 CITATIONS

SEE PROFILE
READS

136
Daniel Kempler

Emerson College

92 PUBLICATIONS 2,413 CITATIONS

SEE PROFILE

Some of the authors of this publication are also working on these related projects:

Project Cortical-subcortical interaction in PD and normal speech View project

Project $\quad$ studies in motor speech View project 


\title{
Prosodic changes in aphasic speech: timing
}

\author{
DIANA VAN LANCKER SIDTIS ${ }^{1}$, DANIEL KEMPLER ${ }^{2}$, \\ CATHERINE JACKSON ${ }^{3}, \&$ E. JEFFREY METTER ${ }^{4}$ \\ ${ }^{1}$ New York University, New York, USA, ${ }^{2}$ Emerson College, Boston, USA, ${ }^{3}$ California State University \\ at Northridge, USA, and ${ }^{4}$ National Institute on Aging, Bethesda, MD, USA
}

(Received 16 May 2009; Accepted 4 November 2009)

\begin{abstract}
Controversy remains about the impairment of prosody in aphasia, particularly with regard to speech timing. This paper addresses this topic through an analysis of timing in four sets of a common morphological paradigm. The paradigm consisted of a basic form (stem) and two longer derived forms (e.g. zip, zipper, zippering). Normally, vowel durations are shorter in longer derived forms (e.g. zippering) than in the stem (e.g. zip), due to a process called 'initial shortening'. Twelve patients with aphasia (four each Broca, Wernicke, and Anomic), and 11 age-matched healthy adults were assessed. Structural (CT) and functional brain imaging (PET) were available for all patients. While all groups showed initial shortening between the stem and the derived forms, the patients with Broca's aphasia presented an inverse pattern between the two derived forms (longer initial vowel in 'zippering' than 'zipper'), and the patients with Wernicke's aphasia produced significantly longer vowel durations overall than the healthy participants. The results are related to radiological information regarding the location of structural and functional brain damage and relative preservation and loss of prosodic features in cerebral damage.
\end{abstract}

Keywords: acoustic analysis, aphasia, prosody, timing

\section{Introduction}

Speech prosody is generally considered to include systematic changes in perceived segment length, loudness, and pitch, corresponding to acoustic variations in time (segment duration), intensity (amplitude), and fundamental frequency $\left(\mathrm{F}_{0}\right)$, respectively. Studies of normal speech have indicated that speech segment lengths and relationships are predictable in specific linguistic and phonetic contexts (House, 1961; Lehiste, 1972; Klatt, 1976; Kent and Forner, 1980). For example, words in final position in a phrase or occurring before a pause are longer than the same word embedded in a phrase, a phenomenon known as 'prepausal lengthening' (Gaitenby, 1965). A related process, known as 'initial shortening', describes the fact that durations of vowels in word stems are shorter when embedded in longer derived forms: i.e. /i/ in stick is longer than the /i/ in sticky or stickily (Lehiste, 1972). These temporal patterns occur reliably in normal speech.

Correspondence: Daniel Kempler, Communication Sciences and Disorders, Emerson College, 120 Boylston St., Boston, MA 02118, USA. Tel: 617-824-8302. E-mail: daniel_kempler@emerson.edu 
Normal speech timing and rhythmic relations have been consistently associated with left hemispheric function (Zatorre and Belin, 2001). Disruption of timing relations has been reported in left-hemisphere (LH) damage associated with dysarthria and apraxia of speech (Whitty, 1964; Darley, Aronson, and Brown, 1975; Kent and Netsell, 1975; DiSimoni and Darley, 1977; Kent, Netsell, and Abbs, 1979; Kent and Rosenbek, 1982; 1983; Collins, Rosenbek, and Wertz, 1983). Indeed, the well-known parameter 'impaired melody of speech' associated with LH damage in the Boston Aphasia Diagnostic system (Goodglass and Kaplan, 1972) actually may involve primary disruption of temporal integrity in spoken output (Danly and Shapiro, 1982; Seddoh, 2004).

The issue of speech timing patterns in patients with aphasia is complex because of the variety of symptoms and syndromes in aphasia as well as the range of timing patterns that can be observed and measured. The impairment or preservation of speech timing in aphasia appears to be determined in part by the type of aphasia and the particular timing pattern investigated.

Danly and Shapiro (1982) investigated several aspects of speech prosody in patients with Broca's aphasia, including sentence terminal $F_{0}$ fall, $F_{0}$ declination, and sentence final lengthening. Their results indicate relative preservation of the $F_{0}$ variations (see also Seddoh, 2004) but absence of sentence-final lengthening. In contrast to healthy speakers, patients with Broca's aphasia showed shorter, rather than the expected longer sentence-final words compared to the same words in sentence initial and medial positions. Similarly, other investigations (e.g. Baum and Boyczuk, 1999; Baum and Pell, 1999; Gandour, Ponglorpisit, Khunadorn, Dechongkit, Boongird, and Satthamnuwong, 2000; Baum, Pell, Leonard, and Gordon, 2001; Shah, Baum, and Dwivedi, 2006) indicate impaired speech timing in both fluent and non-fluent aphasia, but more often seen in non-fluent aphasia (e.g. Seddoh, 2008). Furthermore, non-fluent aphasia appears more likely to disrupt timing in any length units (including monosyllabic words), while disruptions in fluent aphasia appear to be more marked in longer speech units (multisyllabic words, phrases, and sentences) (Baum and Boyczuk, 1999). This has led to speculation that anterior brain damage impairs phonetic implementation in any length linguistic unit, while posterior damage impairs speech planning of longer units (e.g. Balan and Gandour, 1999). Recent studies (e.g. Seddoh, 2004; 2008) compared syntactically conditioned speech timing in patients with fluent and non-fluent aphasia, including timing of sentences in short vs long statements and short vs long questions. Patients with fluent aphasia showed longer absolute durations than healthy speakers, but overall similar patterns and durations to the healthy participants, while patients with nonfluent aphasia exhibited abnormally long durations on all segmental measures. Speech timing disruption in non-fluent aphasia may have multiple origins, with phonetic disruption occurring in short utterances, and syntactic planning problems manifest in phrase and sentence length units.

The work reported here expands the investigation of speech timing in aphasia by examining word-level, morphologically conditioned aspects of speech timing in patients with fluent and non-fluent aphasia. This investigation further explores temporal control in aphasic speech as reflected in the phenomenon of initial shortening in morphologically-related words. The morphological paradigms used in this study were chosen because they show robust initial shortening effects in both healthy and brain-damaged subjects in past research (Lehiste 1972; Wertz, Collins, Weiss, Kurtzke, Friden, Brookshire, et al., 1981).

Normally, in a pattern called 'initial shortening', vowels are longer in word stems than they are in each subsequently shortened derived form (e.g. stick vs sticky vs stickily) (Lehiste, 1972); the stem vowel /i/ is normally longest in the stem stick, shorter in the derived form sticky, and 
still shorter in the tri-syllabic word stickily. Using four sets exemplifying this paradigm, we compared normal values with measures from participants with diagnoses of Broca's aphasia, Wernicke's aphasia, and Anomia. The goal was to determine the extent to which these temporal relationships are disturbed in different types of aphasic speech associated with these different diagnoses. Stem (initial) vowel durations were measured and compared to durations in the two longer, derived forms. We predicted that participants with aphasia would perform differently from healthy adults in initial vowel shortening, and that timing patterns would be associated with particular aphasia syndromes.

\section{Methods}

\section{Participants}

Twelve individuals with aphasia were studied, four each with Broca, Wernicke, or Anomic aphasia. These three types of aphasia were chosen because they constitute classic diagnoses which emerge from diagnostic instruments and parallel the prior literature investigating speech production in non-fluent (Broca's), and fluent (Wernicke's and Anomic) aphasias. Individuals with Broca's aphasia used halting, effortful speech, and were without dysarthria (imprecise articulation, voice disorder). Although, as expected, articulatory disorders formed part of the Broca profile (Bernt and Caramazza, 1980; Blumstein, Cooper, Goodglass, Statlendera, and Gottlieb, 1980; Alexander, Naeser, and Palumbo, 1990; Dronkers, Baldo, Ogar, Wilkins, Ludy, Arevalo, et al., 2009), using criteria from Duffy (1995) none of our participants with Broca's aphasia carried the diagnosis of apraxia of speech. Those with Wernicke's aphasia spoke fluently with occasional use of neologisms. Participants with Anomic aphasia, a milder category of language disturbance, used fluent speech with frequent pausing in the word-finding process. All were male, right-handed, literate, native speakers of Standard American English. Education ranged from 8-14 years, with a mean of 11.9 years; ages spanned from 43-76, with a mean of 61.9; time post-onset of injury ranged from 1-81 months, with a mean of 16 months (see Table I). All participants were administered the Western Aphasia Battery (WAB) (Kertesz, 1982), from which they received an aphasic

Table I. Demographic information for participants with aphasia: age, time post-onset (TPO in months) and education (years).

\begin{tabular}{lllcr}
\hline & Number & Age & TPO (months) & Education \\
\hline Broca's & B1 & 64 & 18 & 12 \\
& B2 & 61 & 9 & 12 \\
& B3 & 43 & 8 & $12+$ \\
& B4 & 59 & 14 & 16 \\
& Average & 56.3 & 12.3 & 13 \\
Wernicke's & W1 & 73 & 1 & 8 \\
& W2 & 63 & 5 & 14 \\
& W3 & 65 & 1 & 14 \\
& W4 & 76 & 32 & 11 \\
Anomic & Average & 67.0 & 9.8 & 9.3 \\
& A1 & 60 & 11 & 12 \\
& A2 & 57 & 7 & 12 \\
& A3 & 57 & 5 & 8 \\
& A4 & 67 & 26 & $12+$ \\
\hline
\end{tabular}


Table II. Performance of participants with aphasia on the Western Aphasia Battery.

\begin{tabular}{|c|c|c|c|c|c|c|c|c|}
\hline & Fluency (10) & Comp (10) & $\operatorname{Rep}(10)$ & Naming (10) & $\begin{array}{c}\text { Reading } \\
(100)\end{array}$ & Writing (100) & Praxis (60) & $\mathrm{AQ}$ \\
\hline \multicolumn{9}{|l|}{ Broca } \\
\hline $\mathrm{B} 1^{\star}$ & 5.0 & 9.1 & 6.6 & 8.2 & 73 & 65 & 56 & 71.8 \\
\hline $\mathrm{B} 2$ & 1.0 & 7.4 & 1.7 & 1.3 & 52 & 24 & 41 & 26.7 \\
\hline B3 & .0 & 8.9 & .7 & 1.1 & 84 & 51 & 59 & 33.4 \\
\hline $\mathrm{B} 4$ & 4.0 & 6.9 & 3.5 & 4.2 & 49 & 25 & 59 & 47.1 \\
\hline Mean & 2.5 & 8.1 & 3.1 & 3.7 & 65 & 41.25 & 53.8 & 44.8 \\
\hline \multicolumn{9}{|l|}{ Wernicke } \\
\hline W1 & 8.0 & 5.7 & 4.5 & 2.4 & 40 & 61 & 47 & 45.1 \\
\hline W2 & 7.0 & 5.9 & 1.5 & .8 & 41 & 13 & 49 & 30.3 \\
\hline W3 & 7.0 & .7 & .3 & .2 & 5 & 0 & 11 & 16.4 \\
\hline W4 & 8.0 & 5.0 & 6.6 & 4.9 & 56 & 4 & 32 & 57.0 \\
\hline Mean & 7.5 & 4.3 & 3.2 & 2.1 & 36 & 19.5 & 34.8 & 37.2 \\
\hline \multicolumn{9}{|l|}{ Anomic } \\
\hline A1 & 5.0 & 9.7 & 7.6 & 7.7 & 72 & 35 & 56 & 74.0 \\
\hline A2 & 9.0 & 10.0 & 9.8 & 9.8 & 84 & 96 & 60 & 97.2 \\
\hline A3 & 6.0 & 8.9 & 9.4 & 7.4 & 84 & 30 & 53 & 79.3 \\
\hline A4 & 6.0 & 10.0 & 8.6 & 9.0 & 88 & 88 & 56 & 85.1 \\
\hline Mean & 8.5 & 76.8 & 88.5 & 10.0 & 82 & 62.25 & 56.3 & 83.9 \\
\hline
\end{tabular}

* Although participant B1 was slightly more fluent and milder than the other participants with Broca's aphasia as well as the WAB diagnostic guidelines, his language output was non-fluent and he clinically presented with relatively typical Broca's aphasia.

classification and an Aphasia Quotient (AQ). The AQ is a measure of the severity of language disability derived from weighted scores based on evaluation of spontaneous speech (information content and fluency), auditory comprehension, word and sentence repetition, and naming performance. Language performance data from the Western Aphasia Battery for participants with aphasia are given in Table II.

Eleven healthy adult participants were selected to be comparable to participants with aphasia in age and gender (all male). All healthy participants were native speakers of American English, born and educated in the US with a mean age of 63.2 years $^{1}$ (range 4775 ), a mean of 13 years of education, and reported no medical history of hearing or speech deficit, neurological or psychiatric disease.

All participants with aphasia underwent structural (CT) and metabolic (PET) radiological studies which demonstrated that all suffered from a single left hemisphere stroke with varying degrees of cortical and sub-cortical structural and metabolic damage. These neuroradiologic studies resulted in nine measures of structural and metabolic damage for each participant. The methods for scanning and rating the brain regions are described in detail in a series of articles by Metter and colleagues (e.g. Metter, Riege, Hanson, Camras, Phelps, and Kuhl, 1984; Kempler, Metter, Jackson, Hanson, Riege, Mazziotta, et al., 1988; Metter, Riege, Hanson, Jackson, Kempler, and Van Lancker, 1988; Metter, Kempler, Jackson, Hanson, Mazziotta, and Phelps, 1989; Metter, Hanson, Jackson, Kempler, Van Lancker, Mazziotta, et al., 1990; Kempler, Curtiss, Metter, Jackson, and Hanson, 1991). The radiologic data highlighted differences between the diagnostic groups: the cohort with Broca's aphasia differed from the others by having more regions of sub-cortical structural damage (i.e. the head of the caudate and thalamus) as well as greater hypometabolism in both inferior and superior frontal areas; 


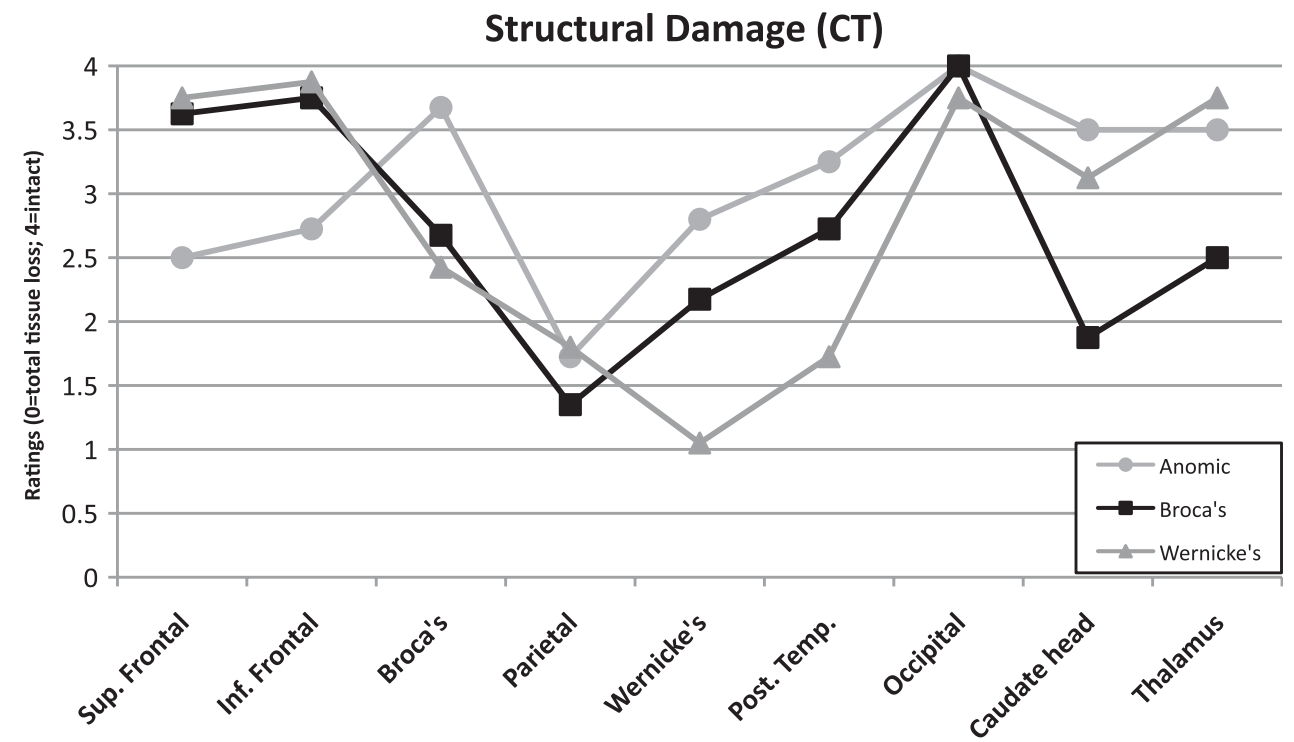

Metabolic Function (PET)

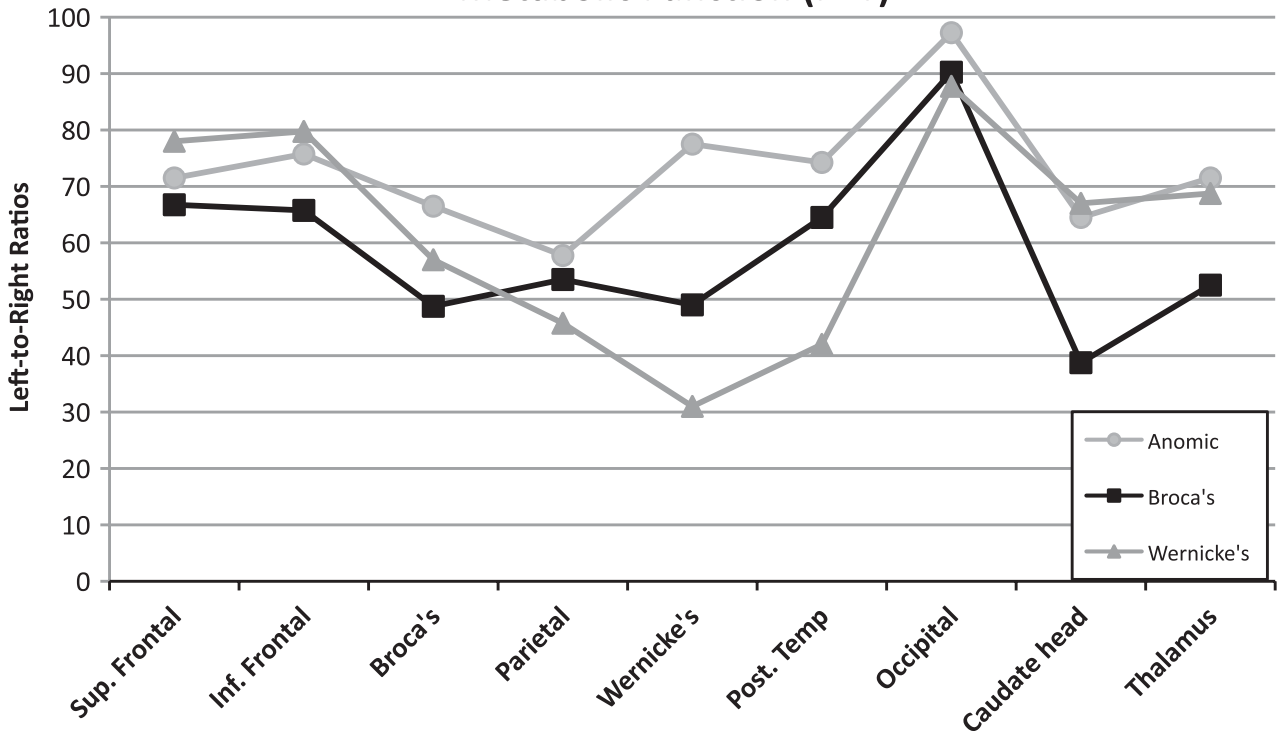

Figure 1. (a) Structural damage (CT). Mean ratings for nine brain regions for each diagnostic group on a five-point scale $(4=$ normal, $3=$ atrophy, $2=$ damage with no tissue loss, $1=$ damage with partial tissue loss, and $0=$ damage with complete tissue loss). (b) Metabolic Function (PET). Metabolic measures for nine brain regions in three patient groups, derived using a comparison of metabolism in homologous regions of the left and right hemisphere for each patient, resulting in a left/right ratio. A higher number indicates greater symmetry between the damaged left hemisphere and the undamaged right hemisphere, and therefore more normal function.

the Wernicke's group showed greater overall structural damage and metabolic asymmetry in Wernicke's area and posterior temporal lobe. The participants with Anomic aphasia showed more prefrontal hypometabolism than the other two groups (see Figures $1 \mathrm{a}$ and $1 \mathrm{~b})$. 


\section{Speech samples}

Stimuli were four three-word paradigms taken from a standard protocol widely used in aphasia research (Wertz et al., 1981), and were similar to those used in the classic study on timing in normal speakers by Lehiste (1972). Speech samples consisted of repetitions of single words: zip, zipper, zippering; flat, flatter, flattering; thick, thicker, thickening; jab, jabber, jabbering. Each repetition was elicited by the examiner using the expression, 'Say __.'. Examiners took care to elicit the stimulus items uniformly across trials and across speakers. Participants who did not produce a satisfactory item on the first trial were asked for a second production. All speakers were able to repeat the full set of stimuli with responses that were suitable for measurement and analysis.

Repetition was used rather than spontaneous speech or reading to minimize the effects of visual reading, language comprehension, and motor planning problems in eliciting audible, intelligible, and segmentable productions. Comparable segmental material was necessary in order to identify and measure vowel length with confidence. Both reading and repetition paradigms elicit productions closer to a typical 'healthy' mean in impaired populations and are associated with higher intelligibility ratings than spontaneous speech (e.g. Snidecor, 1943; Canter and Van Lancker, 1985; Kempler and Van Lancker, 2002). This is likely due to the presence of an external model in the repetition and reading tasks (Georgiou, Iansek, Bradshaw, Phillips, Mattingley, and Bradshaw, 1993; Baev, 1995; Schulz, Greer, and Friedman, 2004). Use of repetition can be expected to minimize differences caused by brain injury. Therefore, any differences present in repetition are more likely to represent robust changes in the system that are not mitigated by the presence of a model (i.e. the stimulus provided by the examiner).

The speech samples were tape-recorded with each subject seated in a sound-treated test room directly in front of a microphone (Electrovoice, Burnsville, MN, Model RE-15) coupled to an Ampex (Redwood City, CA) tape recorder (AG-600) located in an adjacent test room. The mouth-to-microphone distance was 8-inches, and the words were spoken at a comfortable loudness level.

\section{Acoustic analysis procedure}

The vowel nuclei in the stem and two derived forms were measured using a Kay Digital Spectrograph (Model 5500; Kay Elemetrics, Lincoln Park, NJ). To mark the beginning point of the vowel nucleus, cursors were placed by two raters at the beginning of the periodic energy (formants) following the voiceless fricative in thick. For $j a b$ and zip and their derivations, to mark the initial point, the cursor was placed at the first locus of steady state periodic energy following the preceding voiced consonants; similarly for flat, the initial cursor was placed at the first locus of steady state energy following the discontinuous formants for $/ 1 /$. For all four word paradigms, cursors marking the final point of the vowel were placed at the beginning point of the closure for the stem-final consonant. Care was taken that measurement points were consistent across derived forms. Any discrepancies in selection of cursor placement were resolved by agreement between two trained raters.

\section{Results}

Results providing means and standard deviations for vowel nuclei durations from stem and two derivations for all four paradigms are shown in Table III, coded as three 'times' (T), where $\mathrm{TI}=$ stem (e.g. thick) and TII $=$ first derivation (e.g. thicker), and TIII $=$ second 
Table III. Vowel durations. Means (in ms) and standard deviations (SD) for vowel durations in stem syllables (Time I) and derived forms, -er, -ing (Time II and Time III) of the words zip, thick, jab, and flat.

\begin{tabular}{llrrr}
\hline Word & Group & Time I $(S D)$ & Time II $(S D)$ & Time III $(S D)$ \\
\hline Zip & Normal & $75.0(24.4)$ & $60.1(4.6)$ & $77.3(27.7)$ \\
& Wernicke & $190.9(54.8)$ & $130.0(53.3)$ & $121.0(36.0)$ \\
& Broca & $114.4(29.4)$ & $65.2(11.7)$ & $87.5(27.8)$ \\
\multirow{3}{*}{ Thick } & Anomic & $98.4(17.0)$ & $89.8(15.9)$ & $91.0(18.6)$ \\
& Normal & $82.8(11.5)$ & $52.3(7.7)$ & $41.4(9.3)$ \\
& Wernicke & $150.3(35.7)$ & $123.8(51.8)$ & $119.5(61.2)$ \\
& Broca & $99.6(7.4)$ & $68.3(14.5)$ & $78.9(29.4)$ \\
Jab & Anomic & $96.9(26.0)$ & $81.2(23.3)$ & $92.1(32.3)$ \\
& Normal & $296.0(75.9)$ & $135.1(31.4)$ & $126.5(17.0)$ \\
& Wernicke & $266.7(59.9)$ & $185.9(26.3)$ & $185.1(37.3)$ \\
& Broca & $263.2(49.1)$ & $151.1(32.5)$ & $156.6(32.3)$ \\
Flat & Anomic & $283.9(31.8)$ & $180.8(31.2)$ & $183.1(45.7)$ \\
& Normal & $150.8(46.1)$ & $128.1(32.3)$ & $107.0(23.1)$ \\
& Wernicke & $245.6(60.5)$ & $218.3(61.0)$ & $190.6(36.1)$ \\
& Broca & $207.8(41.9)$ & $151.5(57.5)$ & $174.1(64.5)$ \\
& Anomic & $228.4(80.8)$ & $182.8(50.8)$ & $166.7(75.8)$ \\
\hline
\end{tabular}

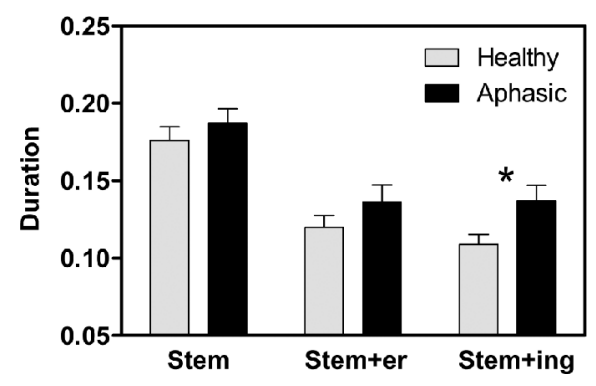

Figure 2. Initial shortening. Comparison of healthy and aphasic speakers across paradigms. Asterisk indicates a significant difference.

derivation (e.g. thickening). Independent samples T-tests compared healthy and aphasic groups on measures of vowels lengths for stem (TI) and the two derived forms (TII, TIII). The two groups (healthy and aphasic) differed only in TIII $(t=-2.27, p=.034)$ (Figure 2). We probed further to examine temporal patterns within aphasic groups. Figure 3 provides these data in graphic format, for visual comparison across words, derived forms, and groups. This figure illustrates clearly that high vowels (zip and thick) are shorter than the low vowels (flat and jab) for all four groups. This pattern, evident in all three groups of aphasic speakers, indicates preservation of the robust phonetic phenomenon of high vowels being shorter than low vowels. It is also clear that the typical finding in healthy participants of vowels being longer before a final voiced consonant (compare flat vs jab) is maintained for all three aphasic groups as well.

A mixed-design analysis of variance (ANOVA) examined the effects of word paradigm (flat. ., jab. .., zip. .., thick. . .) and time (stem, stem + er, stem + er + ing) as repeated measures and diagnostic group as a between subjects variable. An OMNIBUS ANOVA revealed main effects of word paradigm $[F(3,57)=119.82 ; p<.0001$; Eta-squared $=.863]$ and time $[F(2,38)=75.92 ; p<.0001$; Eta-squared $=.800]$, but not diagnosis. However, 


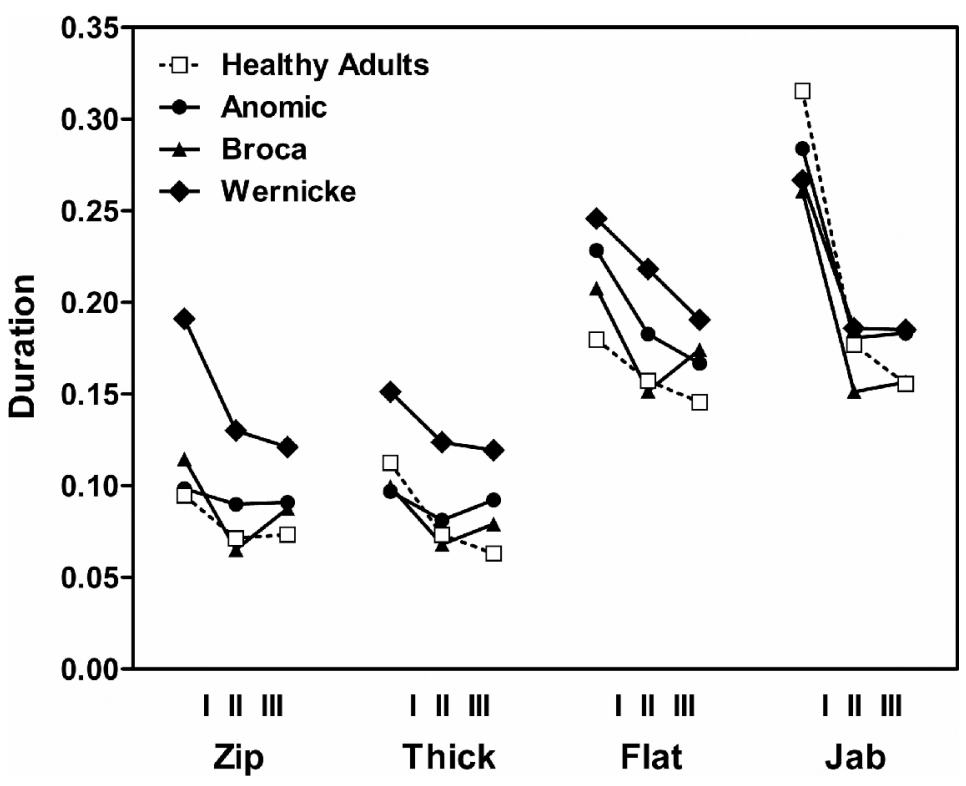

Figure 3. Vowel length. Vowel length in four paradigms and four groups for the stem (I) and two derived forms (II, III), measured in msecs.

diagnosis interacted with word paradigm $[F(9,57)=2.29 ; p<.03 ;$ Eta-squared $=.265]$ and with the interaction between word paradigm and time $[F(18,114)=2.56 ; p=.001$; Etasquared $=.287$ ]. To more directly assess possible differences between the more impaired aphasic sub-groups, the ANOVA was repeated without the Anomic group. This resulted in a main effect of group $[F(2,16)=4.343 ; p=.03]$, a main effect of word paradigm $([F(3,48)=$ $94.127 ; p=.0001]$, and a main effect of time $([F(2,32)=61.744, ; p=.0001]$. In this analysis diagnostic group also interacted significantly with word and time $[F(12,96)=3.283$; $p=$ .001]. The main effect of time in both ANOVAs indicated that speakers produced the vowels with different durations from TI to TII and TIII. Collapsing values over words and times, an ANOVA showed a significant difference between Wernicke and healthy adult values $(F(1,12)=$ $9.01, p<.02)$. This is attributable to the overall longer vowel durations in the Wernicke productions (see Figure 3). The same comparisons made between healthy participants and participants with Broca's and Anomic aphasia were not significant.

To further investigate the main effect of word paradigm, separate 2-way ANOVAs were performed on each of the four word paradigms. This analysis showed significant main effects of group on word paradigms with high (short) vowels: $z i p(F(3,19)=13.225, p<.01)$ and thick $(F(3,19)=3.386, p<.04)$. For words with low (long) vowels ( $j a b$ and flat) the groups were not significantly different. Thus, the group differences are more clearly observed with the high (short) vowel (zip, thick) than the low (long) vowel (jab, flat).

Visual inspection of the average shortening patterns (Figure 3) suggests that all groups produced relatively shorter vowels in TII compared with TI, but differed in the extent and temporal relationship of vowel shortening between TII and TIII. To investigate this further, difference scores were calculated by taking the difference in vowel length between TI (stem) and TII (stem + er) and between TI and TIII (stem + er + ing). When subtracting TI-TII difference from the TI-TIII difference, a positive number indicates further shortening 


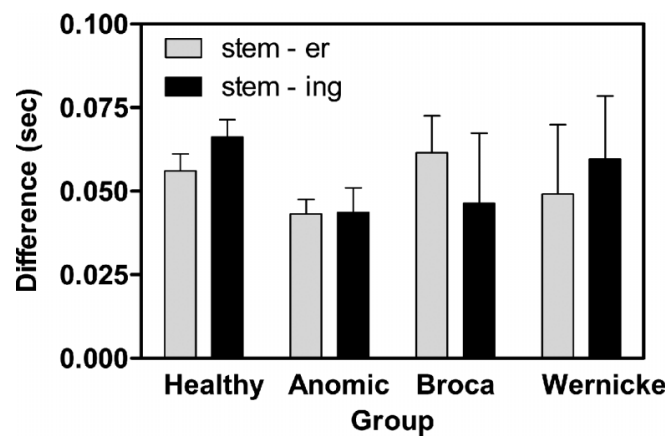

Figure 4. Vowel length difference between stem and derived forms. The difference between vowel lengths at Time I vs Time II compared with Time I vs Time III, for four groups. A higher number indicates greater shortening between the stem vowel and the vowel in the derived form.

between TII and TIII. Two-tailed t-tests comparing the difference scores showed that healthy adults showed the expected further initial vowel shortening between TII and TIII ( $d f=10$, $p=.007$ ). While none of the aphasic groups showed significant shortening between TII and TIII, a unique pattern is notable in the data for the Broca group: Participants with Broca's aphasia showed an inverse pattern of relatively longer TIII than TII initial vowels, thus violating the typical pattern. This difference is supported by an ANOVA on the difference scores (four groups by two difference scores, stem subtracted from stem + er, and stem subtracted from stem + er + ing), which showed a significant group by difference score interaction $(F=(3,19)=3.265, p=.044)$, indicating that the groups are not all following the same pattern (Figure 4 ). While the number of participants is too small to verify specific group differences, graphic rendering of group difference scores shown in Figure 4 suggests that the reversed pattern seen in those with Broca's aphasia accounts for the significant interaction. This pattern has been reported elsewhere (Danly and Shapiro, 1982; Baum, 1992; Ghosh, Tourville, and Guenther, 2008; see Discussion section below).

\section{Discussion}

Different profiles of timing disruptions in a normally robust paradigm were observed in the three aphasic groups. Phonetically, in fluent speech, high vowels (as in /stick/) are typically shorter than low vowels (as in /flat/) and vowels are longer before a voiced consonant (as in / $\mathrm{jab} /$ ) than before a voiceless consonant (as in /flat/). This is described for normal AmericanEnglish speakers (Ladefoged, 2006). These patterns were maintained in the aphasic speakers. While these overall normal phonetic patterns were maintained, profiles of vowel lengths in the morphological paradigm varied with aphasic diagnosis.

The participants with non-fluent (Broca's) aphasia did not produce a typical pattern of shortening of the initial (stem) vowel in several three-word paradigms. Analysis of difference scores from stem (Time I) to Time II and from stem vowel to Time III suggested that the Broca group failed to produce typical vowel shortening, particularly in Time III, the 3-syllable words. To further investigate the abnormal timing patterns in patients with Broca's aphasia, we examined percentage of shortening from Time I to Time II, and noted that none of the individuals with the Anomic diagnosis had shortening greater than normal speakers, while three of the four Broca participants had shortening greater than normal. These observations 
in word paradigms are similar to those made about speech patterns in Broca's aphasia (Danly and Shapiro, 1982) in which pre-pausal lengthening was absent in two and three word utterances. The results are also consistent with results reported by Baum (1992), in which patients with aphasia failed to show decreased root durations in three- as compared to twosyllable words. The present results confirm these earlier findings with additional patient groups in multiple single word paradigms with morphological expansions, documenting consistent motor speech planning problems for patients with non-fluent aphasia.

The patients with Wernicke's aphasia produced longer stem vowels overall compared to the other groups tested. Seddoh (2004) also found longer absolute durations in patients with fluent aphasia. Our results demonstrate that this finding is robust, occurring in a different sample of patients with fluent aphasia and a different, but well-controlled speech task. The slow speech pattern may be attributed to a problem with self-monitoring. An analogous situation can be found in speech under conditions of Delayed Auditory Feedback (DAF). DAF is generally considered to be adverse and disruptive of normal speech, and results in markedly slow speech and prolonged syllable durations (see Lincoln, Packman, and Onslow, 2006 for review). Mysak (1976) postulated that the slowing of speech resulting from DAF may allow a deficient auditory processing system time to perform its control function more efficiently. In DAF, the deficient auditory processing system is created by the DAF system. The slowing of speech rate observed in the Wernicke's patients may reflect the same type of compensatory mechanism, designed to continue speech flow in the presence of a neurologically-based auditory processing impairment. A specific failure in self-monitoring of speech in Wernicke's aphasia has been proposed by others (Marshall, Robson, Pring, and Chiat, 1998).

An interesting observation from visual inspection of the temporal relations data arose from the Anomic patients, whose vowel measurements suggested patterns similar to both the other diagnostic groups. Overall, the Anomic vowel lengths approached those of the Wernicke group (i.e. longer than healthy participants), while their relative shortening of vowels in TII and TIII suggested a pattern similar to those with Broca's aphasia. That is, like the participants with Broca's aphasia, in two out of four of the paradigms, Anomic speakers did not produce a shorter vowel in the final derivation. Further analysis with a larger sample of participants is necessary to pursue the possibility that Anomic subjects show two kinds of abnormal timing patterns.

One goal of investigating speech timing deficits in patients with aphasia is to better understand the neurophysiological basis for speech production in general. Using previously obtained radiological studies, speech timing could be examined with regard to both structural (CT) and functional (PET) damage. Participants with Broca's aphasia, who demonstrated the greatest timing abnormalities, differ from the other groups in the extent of subcortical (caudate and thalamic) structural damage and pre-frontal hypometabolism. This supports both the existence of a frontal-subcortical circuit in speech production (Metter et al., 1988; Sidtis, Strother, and Rottenberg, 2003; Sidtis, Gomez, Groshong, Strother, and Rottenberg, 2006), and the role of pre-frontal regions in motoric control of verbal output (Kurowski, Blumstein, Palumbo, Waldstein, and Burton, 2007). In Wernicke's aphasia, overall speech rate change (slowing) is associated with greater structural and metabolic damage in the temporal lobe. Although frontal cortical function is preserved, it may receive faulty input and impoverished feedback from the temporal lobe. The timing pattern of the participants with Anomic aphasia, similar to the patients with Broca's aphasia, is not surprising given that they share with the Broca's group a significant degree of pre-frontal dysfunction. This highlights again the importance of pre-frontal regions in motor speech planning. Despite the fact 
that there are relatively few participants in each diagnostic group, a coherent picture emerges, wherein the impairment in speech planning occurs to different degrees in different patient groups, but appears to parallel pre-frontal structural and hypometabolic abnormalities. More pervasive timing abnormalities (overall slowing of rate) appear separable and associated with temporal lobe dysfunction.

These findings show timing abnormalities in participants with aphasia, most prominently in patients with non-fluent aphasia. Limitations of this study arise from the number of participants in each of four diagnostic groups and the resultant limit in statistical power and the smaller effect sizes (Eta-squared values) on some of the contrasts, such that expanding group sizes will be necessary to confirm these findings. These results are consistent both with prior literature on speech timing in aphasia (Ghosh et al., 2008) and with the general idea that impaired 'melody of speech' in non-fluent aphasia actually reflects not intonation but timing abnormalities (Sidtis and Van Lancker Sidtis, 2003). While most proposals about hemispheric representation of acoustic components of prosody arise from perception studies, these results provide support for a model of prosodic production that associates pitch control with the right hemisphere and temporal control with the left hemisphere (Alcock, Wade, Anslow, and Passingham, 2000; Zatorre and Belin, 2001).

\section{Acknowledgements}

This research was supported in part by the Intramural Research Program of the NIH, National Institute on Aging. We appreciate the assistance of Andrew Lanto and John J. Sidtis in statistical consulting. We also thank two anonymous reviewers for their comments and Wayne Hanson for his support on this project.

Declaration of interest: The authors report no conflicts of interest. The authors alone are responsible for the content and writing of the paper.

\section{Note}

1. Age for one healthy adult was not available.

\section{References}

Alcock, K. J., Wade, D., Anslow, P., \& Passingham, R. E. (2000). Pitch and timing abilities in adult left-hemispheredysphasic and right-hemisphere subjects. Brain and Language, 75, 47-65.

Alexander, M. P., Naeser, M. A, \& Palumbo, C. (1990). Broca's area aphasias: aphasia after lesions including the frontal operculum. Neurology, 40, 353-362.

Baev, K. V. (1995). Disturbances of learning processes in the basal ganglia in the pathogenesis of Parkinson's disease: a novel theory. Neurology Research, 17, 38-48.

Balan, A., \& Gandour, J. (1999). Effect of sentence length on the production of linguistic stress by left- and righthemisphere-damaged patients. Brain and Language, 67, 73-94.

Baum, S. R. (1992). The influence of word length on syllable duration in aphasia: acoustic analyses. Aphasiology, 6, 501-513.

Baum, S. R., \& Boyczuk, J. P. (1999). Speech timing subsequent to brain damage: effects of utterance length and complexity. Brain and Language, 67, 30-45.

Baum, S. R., \& Pell, M. D. (1999). The neural bases of prosody: insights from lesion studies and neuroimaging. Aphasiology, 23, 581-608.

Baum, S. R., Pell, M. D., Leonard, C. L., \& Gordon, J. K. (2001). Using prosody to resolve temporary syntactic ambiguities in speech production: acoustic data on brain-damaged speakers. Clinical Linguistics and Phonetics, 15, 441-456. 
Berndt, R. S., \& Caramazza, A. (1980). A redefinition of the syndrome of Broca's aphasia: implications for a neuropsychological model of language. Applied Psycholinguistics, 1, 225-278.

Blumstein, S. E., Cooper, W. E., Goodglass, H., Statlendera, S., \& Gottlieb, J. (1980). Production deficits in aphasia: a voice-onset time analysis. Brain and Language, 9, 153-170.

Canter, G. J., \& Van Lancker, D. (1985). Disturbances of the temporal organization of speech following bilateral thalamic surgery in a patient with Parkinson's disease. Fournal of Communication Disorders, 18, 329-349.

Collins, M., Rosenbek, J. C., and Wertz, R. T. (1983). Spectrographic analysis of vowel and word duration in apraxia of speech. Fournal of Speech and Hearing Research, 26, 217-224.

Danly, M., \& Shapiro, B. (1982). Speech prosody in Broca's aphasia. Brain and Language, 16, 171-190.

Darley, F. L., Aronson, A. E., \& Brown, J. R. (1975). Motor speech disorders. Philadelphia: Saunders.

DiSimoni, F. G., \& Darley, F. L. (1977). Effect on phoneme duration control of three utterance-length conditions in an apractic patient. Fournal of Speech and Hearing Disorders, 42, 257-264.

Dronkers, N., Baldo, J., Ogar, J., Wilkins, D., Ludy, C., Arevalo, A., et al. (2009). Lesion localization of chronic aphasia syndromes. Paper presented at the Academy of Aphasia, Boston, 18-20 October.

Duffy, J. R. (1995). Motor speech disorders. Philadelphia: Mosby.

Gaitenby, J. (1965). The elastic word. Status Report on Speech Research, SR-2. New York: Haskins Laboratories.

Gandour, J., Ponglorpisit, S., Khunadorn, F., Dechongkit, S., Boongird, P., \& Satthamnuwong, N. (2000). Speech timing in Thai left- and right-hemisphere damaged individuals. Cortex, 36, 281-288.

Georgiou, N., Iansek, R., Bradshaw, J. L., Phillips, J. G., Mattingley, J. B., \& Bradshaw, J. A. (1993). An evaluation of the role of internal cues in the pathogenesis of parkinsonian hypokinesia. Brain, 116, 1575-1587.

Ghosh, S. S., Tourville, J. A., \& Guenther, F. H. (2008). A neuroimaging study of premotor lateralization and cerebellar involvement in the production of phonemes and syllables. Fournal of Speech, Language E Hearing Research, 51, 1183-1202.

Goodglass, H., \& Kaplan, E. (1972). The assessment of aphasia and related disorders. Philadelphia: Lea and Febiger.

House, A. S. (1961). On vowel duration in English. Fournal of the Acoustical Society of America, 33, 1174-1178.

Kempler, D., Curtiss, S., Metter, E. J., Jackson, C. A., \& Hanson, W. R. (1991). Grammatical comprehension, aphasic syndromes and neuroimaging. Fournal of Neurolinguistics, 6, 301-318.

Kempler, D., Metter, E. J., Jackson, C. A., Hanson, W. R., Riege, W. H., Mazziotta, J. C., et al. (1988). Disconnection and cerebral metabolism. Archives of Neurology, 45, 275-279.

Kempler, D., \& Van Lancker, D. (2002). Effect of speech task on intelligibility in dysarthria: a case study of Parkinson's disease. Brain and Language, 80, 449-464.

Kent, R. D., \& Forner, L. L. (1980). Speech segment durations in sentence recitations by children and adults. Fournal of Phonetics, 8, 157-168.

Kent, R. D., \& Netsell, R. (1975). A case study of an ataxic dysarthric: cineradiographic and spectrographic observations. Fournal of Speech and Hearing Disorders, 40, 115-134.

Kent, R. D., \& Rosenbek, J. C. (1982). Prosodic disturbance and neurologic lesion. Brain and Language, 15, 259291.

Kent, R. D., \& Rosenbek, J. C. (1983). Acoustic patterns of apraxia of speech. Fournal of Speech and Hearing Research, 26, 231-249.

Kent, R. D., Netsell, R., \& Abbs, J. H. (1979). Acoustic characteristics of dysarthria associated with cerebellar disease. Fournal of Speech and Hearing Research, 22, 627-648.

Kertesz, A. (1982). Western Aphasia Battery. New York: Grune and Stratton.

Klatt, D. H. (1976). Linguistic uses of segmental duration in English: acoustic and perceptual evidence. Fournal of the Acoustical Society of America, 59, 1208-1221.

Kurowski, K. M., Blumstein, S. E., Palumbo, C. L., Waldstein, R. S., \& Burton, M. W. (2007). Nasal consonant production in Broca's and Wernicke's aphasics: speech deficits and neuroanatomical correlates. Brain E Language, 100, 262-275.

Ladefoged, P. (2006). A course in phonetics. $5^{\text {th }}$ ed. Boston: Wadsworth.

Lehiste, I. (1972). The timing of utterances and linguistic boundaries. Fournal of the Acoustical Society of America, 51, 2018-2024.

Lincoln, M., Packman, A., \& Onslow, M. (2006). Altered auditory feedback and the treatment of stuttering: a review. Fournal of Fluency Disorders, 2, 71-89.

Marshall, J., Robson, J., Pring, T., \& Chiat, S. (1998). Why does monitoring fail in jargon aphasia? Comprehension, judgment, and therapy evidence. Brain and Language, 63, 79-107.

Metter, E. J., Hanson, W. R., Jackson, C. A., Kempler, D., Van Lancker, D., Mazziotta, J. C., et al. (1990). Temporoparietal cortex in aphasia: evidence from Positron Emission Tomography. Archives of Neurology, 47, 1235-1238. 
Metter, E. J., Kempler, D., Jackson, C., Hanson, W. R., Mazziotta, J. C., \& Phelps, M. E. (1989). Cerebral glucose metabolism in Wernicke's, Broca's and conduction aphasia. Archives of Neurology, 46, 27-34.

Metter, E. J., Riege, W. H., Hanson, W. R., Camras, L. R., Phelps, M. E., \& Kuhl, D. E. (1984). Correlations of glucose metabolism and structural damage to language function in aphasia. Brain and Language, 21, $187-207$.

Metter, E. J., Riege, W. H., Hanson,W. R., Jackson, C. A., Kempler, D., \& Van Lancker, D. (1988). Subcortical structures in aphasia: an analysis based on (F-18)-Fluorodeoxyglucose, Positron Emission Tomography, and Computed Tomography. Archives of Neurology, 45, 1229-1234.

Mysak, E. D. (1976). Pathologies of the speech systems. Baltimore, MD: Williams \& Wilkins.

Schulz, G. M., Greer, M., \& Friedman, W. (2004). The effects of pallidotomy surgery on sentence measures across three tasks in Parkinson patients. Fournal of Medical Speech-Language Pathology, 12, 195-205.

Seddoh, S.A. K. (2004). Prosodic disturbance in aphasia: speech timing versus intonation production. Clinical Linguistics $\mathcal{E}$ Phonetics, 18, 17-38.

Seddoh, S.A. K. (2008). Conceptualisation of deviations in intonation production in aphasia. Aphasiology, 22, 1294-1312.

Shah, A. P., Baum, S. R., \& Dwivedi, V. D. (2006). Neural substrates of linguistic prosody: evidence from syntactic disambiguation in the productions of brain-damaged patients. Brain and Language, 96 78-89.

Sidtis, J. J., Gomez, C., Groshong, A., Strother, S. C., \& Rottenberg, D. A. (2006). Mapping cerebral blood Xow during speech production in hereditary ataxia. NeuroImage, 31, 246-254.

Sidtis, J. J., Strother, S. C., \& Rottenberg, D. A. (2003). Predicting performance from functional imaging data: methods matter. NeuroImage, 20, 615-624.

Sidtis, J. J., \& Van Lancker-Sidtis, D. (2003). A neurobehavioral approach to dysprosody. Seminars in Speech and Language, 24, 93-105.

Snidecor, J. (1943). A comparative study of the pitch and duration characteristics of impromptu speaking and oral reading. Speech Monographs, 10, 50-56.

Wertz, R. T., Collins, M., Weiss, D., Kurtzke, J., Friden, T., Brookshire, R., et al. (1981). Veterans administration cooperative study on aphasia: a comparison of individual and group treatment. fournal of Speech and Hearing Research, 24, 580 .

Whitty, C. W. (1964). Cortical dysarthria and dysprosody of speech. Fournal of Neurological and Neurosurgical Psychiatry, 27, 507-510.

Zatorre, R. J., \& Belin, P. (2001). Spectral and temporal processing in human auditory cortex. Cerebral Cortex, 11, 946-953. 